\section{A) Check for updates}

Cite this: Food Funct., 2018, 9, 3173

\title{
Hibiscus and lemon verbena polyphenols modulate appetite-related biomarkers in overweight subjects: a randomized controlled trial
}

\author{
Marina Boix-Castejón, (D) † ${ }^{\mathrm{a}}$ María Herranz-López, (D)*†b Alberto Pérez Gago, ${ }^{a}$

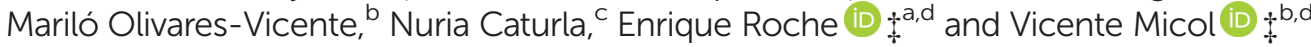

Trial design: Plant-derived polyphenols have shown potential to alleviate obesity-related pathologies by a multi-targeted mechanism in animal models and human intervention studies. A dietary supplement based on a combination of Lippia citriodora (LC) and Hibiscus sabdariffa (HS) polyphenolic extracts was assayed in a double blind and placebo-controlled intervention study with 54 overweight subjects. Methods: Blood pressure, body weight, height, triceps, biceps and abdominal skinfold thickness, and arm and abdominal circumferences were taken at the baseline, 30 and 60 days of the intervention period. The validated Visual Analogue Scale used to record hunger and satiety-related sensations was passed the beginning and at 15 , 30, 45 and 60 days of the intervention. Subjective health status was assessed through the validated SF-36 questionnaire at the beginning and end of the study. Finally, plasma from fasting blood samples was obtained at the beginning, 30 and 60 days of the study. Results: The results showed an improvement of anthropometric measurements, decreased blood pressure and heart rate and a more positive perception in the overall health status. We also observed that plant polyphenols increased anorexigenic hormones (glucagon-like peptide-1) and decreased orexigenic hormones (ghrelin). Conclusions: Based on previous evidence we postulate that AMP-activated protein kinase may have a role in such effects through its capability to modulate energy homeostasis, total daily energy expenditure and lipid management. Although further research may be required, we propose that this polyphenolic combination may be used for weight management by increasing long-term weight loss maintenance through the modulation of appetite biomarkers. This may help to avoid the undesired weight regain typical of calorie restriction diets.

Received 23rd February 2018, Accepted 30th April 2018

DOI: $10.1039 / \mathrm{c} 8 \mathrm{fo} 00367 \mathrm{j}$

rsc.li/food-function known as metabolic syndrome. There seem to be three interconnected reasons for obesity to onset: hyperphagy, caloric imbalance (excess food intake with low energy expenditure) and activation of cell mechanisms to favor excess energy storage as fat. ${ }^{2-4}$

Obesity is generally treated with a combination of approaches, including adopting a dietary intervention focused on calorie restriction, exercise and psychological support in order to achieve the real goals of weight reduction and efficient metabolic control of the pathology. ${ }^{5,6}$ However, these strategies are difficult to implement in the overweight population in the long-term, where the patients tend to abandon treatment and regain the lost weight. As a result, obesity as well as the related noncommunicable diseases are common and often go unnoticed and untreated.

In weight stable obesity, energy intake and expenditure are balanced. As a result of an energy-restricted diet, the subsequent energy deficit and weight loss lead to hunger and reduced energy expenditure. This discordance between appetite and energy expenditure is named the "energy gap" and 
leads to a body metabolic adaptation focused to regain the lost weight. Peripheral signals of energy and nutrient deprivation are sent to the hypothalamus, which increases orexigenic hormones such as ghrelin (oriented to induce hunger), while reducing the contribution of the components of the total daily energy expenditure (TDEE), such as the resting metabolic rate (RMR) (burn less calories when inactive), the thermic effect of food (energy expenditure in response to food ingestion) and thermogenesis. ${ }^{7-10}$ Then, dietary or lifestyle strategies focused on promoting less hunger and greater satiation should be adopted to minimize weight regain.

Obesity is a complex pathology with many factors involved, including the presence of chronic, low-level inflammatory components, altered energetic metabolism and an oxidative imbalance. ${ }^{11}$ Such complexity is one of the reasons why many times it is necessary to include alternative approaches in the weight loss intervention, such as bariatric surgery, pharmacology, and nutritional intervention with dietary supplements or nutraceuticals. $^{6}$ All these approaches are part of the "Global Action Plan for the Prevention and Control of Noncommunicable Diseases 2013-20" developed by the WHO to fight global obesity.

Emerging scientific evidence indicates that dietary supplements may become an alternative for the obesity management and other metabolic disorders. Recent in vitro studies suggest that polyphenolic extracts from Lippia citriodora (LC) and Hibiscus sabdariffa (HS) reduce intracellular lipid accumulation and decrease high glucose-induced oxidative stress and inflammation in adipose tissue through the regulation of different metabolic pathways. ${ }^{12-17}$ The accumulated evidence suggests that a multi-targeted mechanism (regulation of signaling and energy-sensitive pathways, oxidative stress and inflammation-related processes, mitochondrial functionality and membrane-dependent processes) is involved rather than the simple modulation of oxidative stress. ${ }^{17-20}$ Most of these effects have been corroborated in hyperlipidemic animal models in which the continuous consumption of these polyphenolic extracts prevented fatty liver disease and improved lipid metabolism. The blood pressure lowering effects have also been corroborated in patients with metabolic syndrome. ${ }^{14}$

Furthermore, a nutraceutical product, which combined LC and HS polyphenolic extracts with in vitro AMP-activated protein kinase (AMPK)-activating properties, ${ }^{13,16}$ was developed to explore its potential use in alleviating obesity-related pathologies. Therefore, this intervention study was developed with the objective of assessing anthropometric and biochemical parameters in connection with satiety measurements in order to explain the observed effects. This was performed using validated questionnaires and analyzing circulating hunger- and satiety-related hormones and peptides.

\section{Materials and methods}

\section{Supplement formulation}

MetabolAid® (Patent application number P201731147) capsules were kindly provided by Monteloeder SL (Elche, Alicante,
Spain). MetabolAid® contains an optimized combination of polyphenolic extracts from Hibiscus sabdariffa and Lippia citriodora, with a final content of $3.5 \%$ anthocyanins and $15 \%$ verbascoside (\% dry weight, w/w) as measured by high performance liquid chromatography coupled with mass spectrometry detection. $^{12}$

\section{Subjects}

Participants were recruited from a Dietetics Shopping Centre in Elche (Alicante, Spain) during 2016. The participant flow diagram is indicated in Fig. 1. Using the inclusion criteria (females with a body mass index, BMI, 25-34.9 $\mathrm{kg} \mathrm{m}^{-2}$ ), 70 prospective participants responded to the initial recruitment. Exclusion criteria included presence of any obesity-related pathology, use of prescribed medication for hypercholesterolemia or hypertension, consumption of antioxidant supplements/drugs, frequent alcohol consumption and being pregnant/lactating. Based on the above criteria, 54 eligible participants, aged 30-75 years old, passed a telephone-based health screening. The assignment of women to the different groups was carried out by the research team using sequentially numbered containers.

Random distribution as measured by BMI gave the following figures: L1-experimental group $(n=25$; BMI $=29.84)$ with 14 overweight and 11 obese, and L2-placebo group ( $n=22$; $\mathrm{BMI}=29.95)$ with 12 overweight and 10 obese. Since the average BMI of the participants under study was 29.9 and 26 participants were overweight, as a way of simplification, throughout the manuscript the term overweight will be used to refer weight status suffered by the average of the participants.

Prior to participating in the study, subjects were informed by the investigators about the product and the study procedures. All subjects provided a written informed consent before participating that was approved by the Ethics Committee of Miguel Hernández University (reference IB.

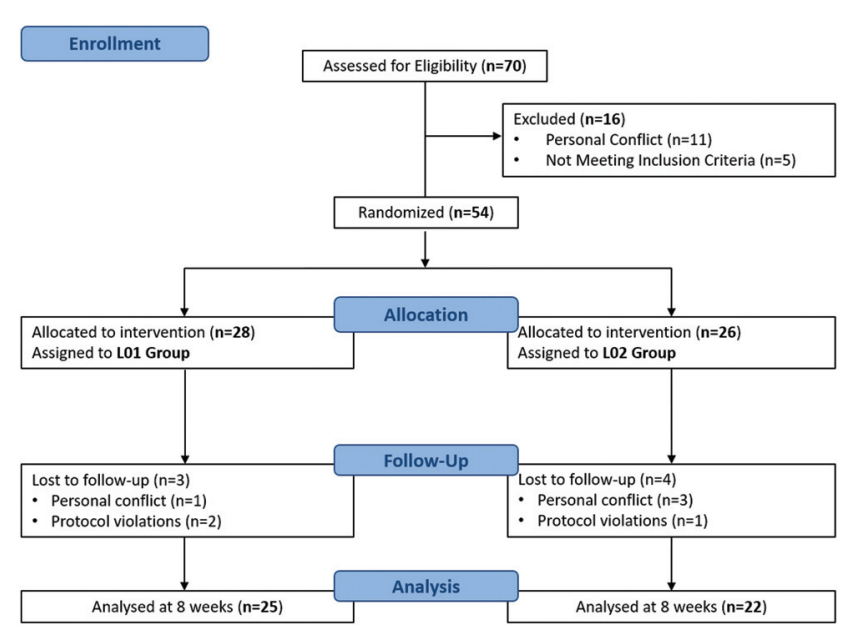

Fig. 1 Participant flow diagram. L1 corresponds to the dietary supplement group and $L 2$ to the placebo group. 
ER.01.15). In addition, the study was conducted in accordance to the Helsinki Declaration (1983 version).

\section{Trial design}

The study was an 8-week, randomized, double blind (participants and field researchers did not know the type of capsules assigned), placebo-controlled trial. After recruitment, subjects were randomly assigned into the L2-placebo $(n=26)$ or L1experimental group $(n=28)$ in a $1: 1$ ratio using the Excel program (Fig. 1). The L1 group (mean age 51) received one capsule containing $500 \mathrm{mg}$ of LC (35\%)-HS (65\%) per day. The L2 group (mean age 51) received one capsule daily of placebo (500 mg of crystalline microcellulose). MetabolAid ${ }^{\circledR}$ and placebo capsules were prepared so that they were the same size, odor and color. Trial participants were instructed to take one capsule 20-30 min prior to breakfast every day for two months.

Throughout both prior and post intervention periods, all participants completed a validated semiquantitative food frequency questionnaire and a 24-hour diet recall. Data showed that the trial participants did not follow a balanced, varied and complete diet. To calculate the energy requirements of each participant, the sedentary life of the subjects was taken into account. The dietary patterns were isocaloric diet equal in total energy (2200 kcal per day energy intake), energy density, dietary fiber and macronutrient with normal hydration. Participants were instructed by a qualified dietician to walk for at least 30 minutes per day.

Compliance of the subjects with the ingestion of capsules and diet was assessed at each clinic visit or by telephone interviews every week. Measurements were taken at the beginning and after 30 and 60 days of the study, unless otherwise stated. A total of 3 and 4 participants were excluded from L2 and L1 groups, respectively (Fig. 1).

\section{Intervention}

Secondary outcome measures include anthropometric determinations, blood pressure measurements and completion of validated questionnaires (VAS and SF-36). Anthropometric measurements were taken at the baseline, 30 and 60 days of the intervention period, and included body weight, height, triceps, biceps and abdominal skinfold thickness, and arm and abdominal circumferences. Abdominal circumferences (AC) were measured at two different sites: anteriorly midway between the xiphoid process of the sternum and the umbilicus and laterally between the lower end of the rib cage and iliac crests (AC1) and at the umbilicus level (AC2). Body weight and height were measured using a scale with a height-measuring rod. Body mass index (BMI) was derived from body weight and height using the equation BMI = body weight $(\mathrm{kg}) /$ height $^{2}(\mathrm{~m})$. Skinfold thickness and circumferences were measured using a skinfold caliper and metric tape, respectively. Body fat percentage was derived from AC1 and AC2 perimeters using the Weltman equation. ${ }^{21}$ In addition, resting systolic and diastolic blood pressures and heart rate were measured at the beginning, 30 and 60 days of the intervention using an Ecomed blood pressure monitor (Medisana Healthcare SL, Barcelona, Spain).

The validated Visual Analogue Scale (VAS) was used to record hunger, satiety, fullness, prospective food consumption, desire to eat something fatty, salty, sweet or savory, and palatability of meals. VAS was passed at rest at the beginning and 15, 30, 45 and 60 days of the intervention. In addition, to assess the individual's subjective health status, patients completed the validated SF-36 questionnaire at the beginning and end of the study (Optum ${ }^{\mathrm{TM}}$ SF-36v2 ${ }^{\circledR}$ Health Survey). The SF-36 Health Questionnaire is composed of 36 items and assesses both positive and negative health status. The 36 items cover the following scales: general health, physical function, physical role, body pain, vitality, social function, emotional role and mental health. Higher scores of SF-36 correspond to the optimal health perception in each item.

Finally, blood samples were obtained from the antecubital vein in EDTA vacutainers after overnight fasting at the beginning, 30 and 60 days of the study. Plasma was isolated immediately by standard procedures ${ }^{22}$ and samples were stored at $-80{ }^{\circ} \mathrm{C}$ until the moment of measurement.

\section{Peptides and circulating hormones}

Primary outcome measures include satiety-related peptides. Plasma was extracted from subjects randomly assigned to the Supplement (L1) or Placebo (L2) group. The Immunoassay kit ProcartaPlex® of the eBioscience division of Affymetrix (multiple immunoassay for convenience and Mix \& Match panels) was used to determine the levels of FGF-23, glucagon-like peptide-1 (GLP-1), ghrelin, insulin, leptin, C-peptide, PYY and resistin. To measure the levels of the abovementioned appetite and satiety markers the Luminex analyzer (Thermo Fisher Scientific) was used.

The changes in the different parameters and hormones related to the circulating satiety-related peptides at the beginning of the study and at 60 days were analyzed. The statistical study was carried out with the help of the software program Graphpad Prism, evaluating the evolution of the parameters studied and comparing the value at the beginning of the study with those obtained at the end of the study for the same individual.

\section{Theoretical estimation of sample size}

As we were interested as a primary outcome in detecting changes in circulating satiety-related peptides within each group after 2 months following the polyphenol extract intake, we considered an effect size $(d=1.53)$ with a two-sided $5 \%$ significance level and a power of $80 \%$. This allowed obtaining a minimal sample size of around 8 participants per group.

\section{Stopping guidelines}

The only rule considered to stop the study was related with the valid number of observations needed to obtain statistically significant results. In addition, a particular participant could be excluded from the study, once included in the trial, for the following reasons: protocol violations, personal reasons and pregnancy. 


\section{Statistical analysis}

Statistical analysis was carried out using the Graphpad Prism software. Results were expressed as means \pm standard deviation (SD). Anthropometric and vital signs (inter-group), VAS and SF-36 data were analyzed using Student's unpaired $t$-test. By contrast, anthropometric and vital signs data (intra-group statistical) and satiety peptides and hormones were analyzed using Student's paired $t$-test. Outcome variables were assessed for conformance to the normal distribution and transformed if necessary by a KS-test. Reported $p$-values were two-sided and values of 0.05 or less $(0.01,0.001$ and 0.0001$)$ were considered statistically significant for comparisons to the baseline (beginning of the study) and between groups (L1 vs. L2) at the same time point of the study. The results of the SF-36 questionnaire were analyzed using the Health Outcomes Scoring Software.

\section{Results}

\section{Anthropometric and vital parameters}

Baseline characteristics of the two groups were well matched and no significant differences were detected at the beginning of the study in the Supplement (L1) vs. Placebo (L2) groups. Participants were recruited from February to March 2016 and the intervention occurred during April-May 2016, with a follow-up at the end of April 2016. The results indicated an overall improvement of the anthropometric data in the L1 group compared to L2 after two months, especially regarding body weight, triceps skinfold thickness, body fat and hip circumference. All parameters decreased after the two-month intervention period. The L1 group presented a higher decrease of triceps skinfold thickness compared to the control group $(-0.15 \pm 0.15 \mathrm{~cm}$ in L2 vs. $-1.64 \pm 0.29 \mathrm{~cm}$ in $\mathrm{L} 1, p<0.001)$
(Table 2). Hip circumference decreased over time in both groups, although the effect was more pronounced in the L1 group $(-3.50 \pm 0.37 \mathrm{~cm})$ compared to the placebo group $(-1.30 \pm 0.28 \mathrm{~cm}, p<0.0001)$. Consistent with this data, AC2 was reduced by $2.57 \pm 0.34 \mathrm{~cm}$ in the $\mathrm{L} 1$ group, as opposed to only $0.80 \pm 0.55 \mathrm{~cm}$ in the L2 group, $p<0.01$. Arm circumference also exhibited a significant decrease compared to the placebo group $(-0.22 \pm 0.23 \mathrm{~cm}$ in $\mathrm{L} 2$ vs. $-0.32 \pm 0.08 \mathrm{~cm}$ in L1, $p<0.001$ ). Body fat decreased over time in both groups but the experimental group (L1) lost significantly more body fat $(-0.83 \pm 0.08 \%)$ compared to the placebo group $(-0.45 \pm$ $0.07 \%$ ) (Table 2).

In addition, significant differences were detected regarding heart rate and blood pressure parameters between the group taking the supplement and the placebo group. A significant decrease was observed in the heart rate of subjects in the L1 group after 60 days, evolving from 73.3 to 68.6 beats per minute (bpm) (Table 1). Also, systolic and diastolic blood pressures significantly decreased in the L1 group after 60 days. Specifically, systolic blood pressured dropped from $117.4 \mathrm{mmHg}$ to below $113.9 \mathrm{mmHg}$, while diastolic pressure went from $73.4 \mathrm{mmHg}$ to below $69.5 \mathrm{mmHg}$ (Table 1).

\section{Appetite assessment}

The scores for hunger, satiety, fullness and prospective food consumption are shown in Fig. 2 for both groups. The data show statistically significant differences between the two groups and at the different time points $(15,30,45$ and 60 days). In the Visual Analogue Scales analysed, statistically significant differences were seen with regard to feelings of appetite, hunger and satiation in the group having the supplement compared to placebo after 1 month, these differences being greater after 45 days and 2 months of product consumption. According to the obtained scores, the consumption of the poly-

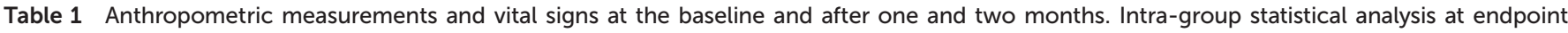
compared to the baseline expressed as means \pm SD. Significance was established at: ${ }^{*} p<0.05,{ }^{\star *} p<0.01,{ }^{\star * \star} p<0.001,{ }^{\star * \star *} p<0.0001$

\begin{tabular}{|c|c|c|c|c|c|c|}
\hline \multirow[b]{2}{*}{ Anthropometric parameters } & \multicolumn{3}{|l|}{ Placebo (L2) } & \multicolumn{3}{|c|}{ Supplement (L1) } \\
\hline & Baseline & Month 1 & Month 2 & Baseline & Month 1 & Month 2 \\
\hline Body weight (kg) & $75.64 \pm 12.92$ & $74.20 \pm 12.62^{* * * *}$ & $73.56 \pm 12.57^{* * * *}$ & $75.26 \pm 9.06$ & $72.80 \pm 9.45^{* * * *}$ & $71.78 \pm 9.06^{* * * *}$ \\
\hline Body mass index $\left(\mathrm{kg} \mathrm{m}^{-2}\right)$ & $29.78 \pm 4.19$ & $29.15 \pm 4.08^{* * * *}$ & $28.95 \pm 4.01^{* * * *}$ & $29.60 \pm 3.40$ & $28.60 \pm 3.52^{* * * *}$ & $28.26 \pm 3.46^{* * * *}$ \\
\hline Arm circumference $(\mathrm{cm})$ & $31.20 \pm 3.80$ & $31.23 \pm 3.80$ & $30.98 \pm 4.10$ & $30.58 \pm 1.67$ & $30.47 \pm 1.83$ & $30.26 \pm 1.76^{* * * *}$ \\
\hline $\mathrm{AC} 1(\mathrm{~cm})$ & $94.02 \pm 13.03$ & $92.84 \pm 12.86^{* * *}$ & $92.05 \pm 13.16^{* * * *}$ & $90.96 \pm 9.03$ & $88.96 \pm 9.18^{* * * *}$ & $88.01 \pm 8.90^{* * * *}$ \\
\hline $\mathrm{AC} 2(\mathrm{~cm})$ & $100.7 \pm 14.01$ & $100.5 \pm 14.21$ & $99.90 \pm 14.54^{* *}$ & $96.42 \pm 7.93$ & $94.84 \pm 7.87^{* * * *}$ & $93.85 \pm 7.95^{* * * *}$ \\
\hline Hip circumference $(\mathrm{cm})$ & $108.8 \pm 8.73$ & $108.15 \pm 8.55^{* *}$ & $107.5 \pm 8.48^{* * *}$ & $110.4 \pm 7.23$ & $108.2 \pm 7.56^{* * * *}$ & $106.9 \pm 7.48^{* * * *}$ \\
\hline Triceps skinfold thickness (mm) & $43.25 \pm 9.28$ & $43.15 \pm 9.34$ & $43.10 \pm 9.42$ & $41.62 \pm 8.18$ & $40.96 \pm 8-08^{* *}$ & $39.98 \pm 7.97^{* * * *}$ \\
\hline Biceps skinfold thickness (mm) & $41.53 \pm 14.46$ & $41.25 \pm 14.35^{*}$ & $41.09 \pm 14.35^{*}$ & $38.33 \pm 10.63$ & $37.61 \pm 10.87^{*}$ & $36.87 \pm 10.52^{* * * *}$ \\
\hline $\begin{array}{l}\text { Abdominal skinfold } \\
\text { thickness (mm) }\end{array}$ & $35.72 \pm 11.15$ & $35.39 \pm 10.68$ & $34.39 \pm 10.68$ & $41.45 \pm 12.89$ & $39.72 \pm 12.95^{* * *}$ & $38.60 \pm 13.08^{* * *}$ \\
\hline$\%$ Body fat & $44.98 \pm 2.71$ & $44.69 \pm 2.65^{* * *}$ & $44.53 \pm 2.64^{* * * *}$ & $44.66 \pm 2.04$ & $44.10 \pm 2.10^{* * * *}$ & $43.83 \pm 2.06^{* * * *}$ \\
\hline \multicolumn{7}{|l|}{ Vital signs } \\
\hline Heart rate (bpm) & $71.41 \pm 8.89$ & $71.95 \pm 8.85$ & $72.09 \pm 9.24$ & $73.32 \pm 9.70$ & $70.84 \pm 7.54^{*}$ & $68.64 \pm 7.07^{* * * *}$ \\
\hline Systolic pressure (mm Hg) & $114.5 \pm 23.26$ & $115.1 \pm 23.55^{*}$ & $115.2 \pm 23.13^{*}$ & $117.4 \pm 12.13$ & $115.1 \pm 12.82^{* *}$ & $113.9 \pm 12.5^{* * * *}$ \\
\hline Diastolic pressure (mm Hg) & $73.73 \pm 10.57$ & $74.05 \pm 11.50$ & $73.73 \pm 10.70$ & $73.40 \pm 5.00$ & $71.28 \pm 5.89^{* * * *}$ & $69.48 \pm 6.62^{* * * *}$ \\
\hline
\end{tabular}

Abbreviations used: bpm, beats per minute. 
A

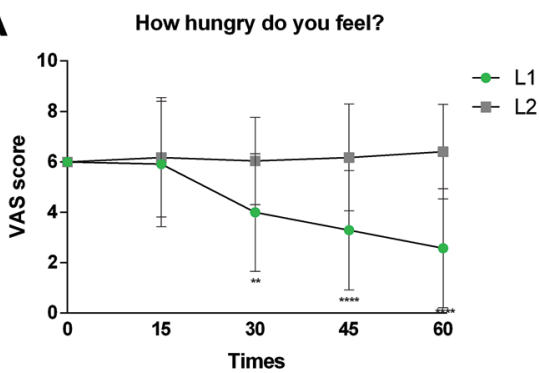

C

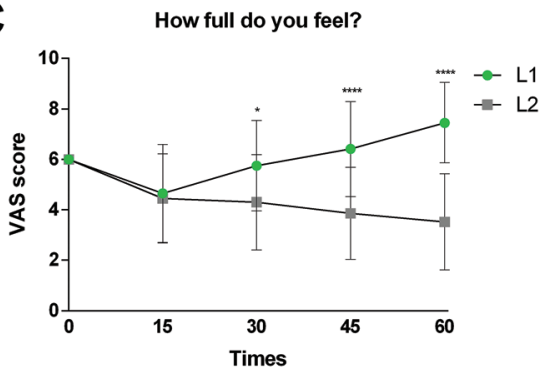

E

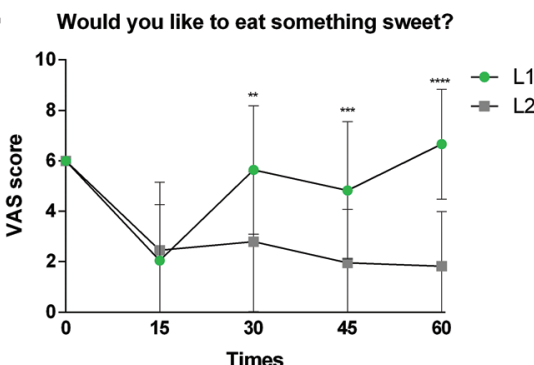

G

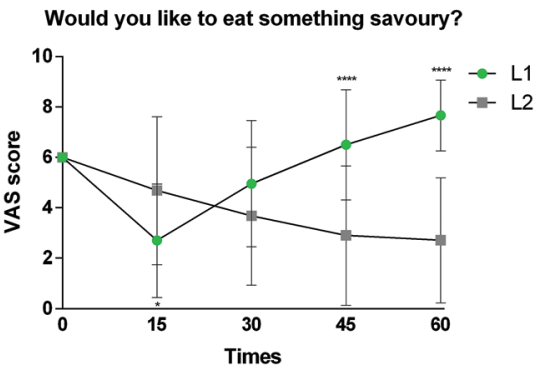

B

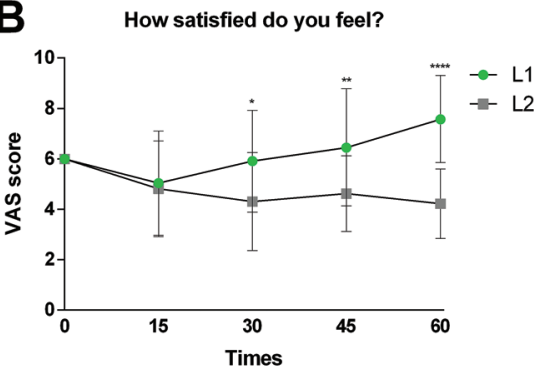

D How much do you think you can eat?

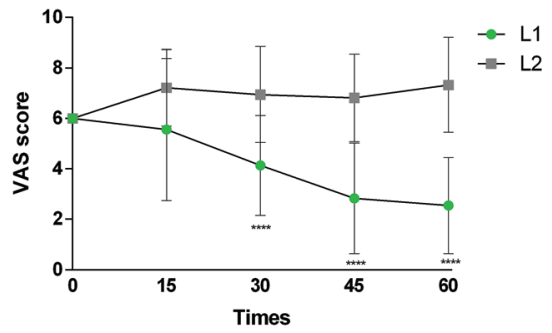

F

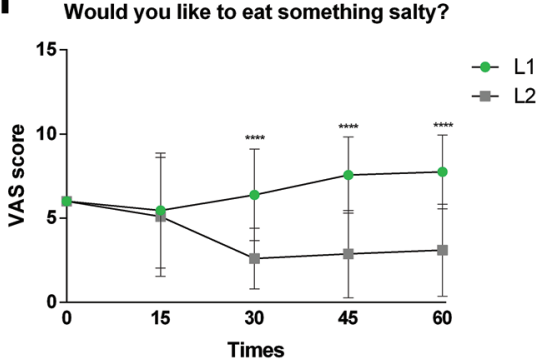

H

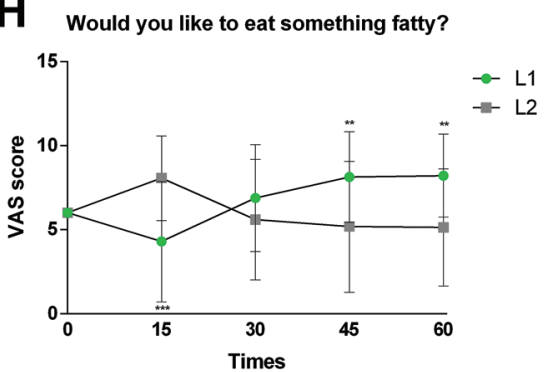

Fig. 2 Subjective scores of hunger-related sensations in the dietary supplement group (L1, $n=25)$ and the placebo group (L2, $n=22)$ after 15,30 , 45 and 60 days, expressed as means \pm SD. Data represent scores of each item compared to placebo. Statistically significant differences were established at: ${ }^{*} p<0.05,{ }^{* \star} p<0.01,{ }^{* \star *} p<0.001,{ }^{* \star * \star} p<0.0001$. Item scores: 1 . How hungry do you feel?: 0 "I am not hungry at all" -10 "I have never been more hungry". 2. How satisfied do you feel?: 0 "I am completely empty" - 10 "I cannot eat another bite". 3. How full do you feel?: 0 "Not at all full" - 10 "Totally full". 4. How much do you think you can eat?: 0 "Nothing at all" - 10 "A lot". 5. Would you like to eat something sweet?: 0 "Yes, very much" - 10 "No, not at all". 6. Would you like to eat something salty?: 0 "Yes, very much" - 10 "No, not at all". 7. Would you like to eat something savoury?: 0 "Yes, very much" - 10 "No, not at all". 8. Would you like to eat something fatty?: 0 "Yes, very much" - 10 "No, not at all".

phenolic supplement significantly decreased the feeling of hunger consistently throughout the two months of intervention (Fig. 2A). The average hunger feeling decreased from 5.92 (day 15) to 2.58 (day 60) in the L1 group, whereas an increase from 6.18 (day 15) to 6.41 (day 60) was observed for the placebo group.
Furthermore, satiety increased from 5.04 (day 15) to 7.58 (day 60) in the intervention group, while it decreased from 4.82 (day 15) to 4.22 (day 60) in the placebo group (Fig. 2B). In line with this result, an increase was also observed in the answer to the question "How full do you feel?" in the intervention group (from 4.65 to 7.46 at day 60 ) compared to the 
placebo group, where it modestly decreased (from 4.45 to 3.52 at day 60) (Fig. 2C).

After two months of intervention, a much lower score was also confirmed in response to question no. 4 "How much do you think you can eat?" in group L1 (from 5.57 to 2.54 at day 60) compared to the placebo group (from 7.22 to 7.33 at the 60) (Fig. 2D). Finally, participants in the control group showed greater preferences for sweet, salty, savoury and fatty foods, questions 5 to 8 , compared to those consuming the polyphenolic supplement (Fig. 2E-H).

\section{SF-36 questionnaire assessment}

After evaluating the SF-36 questionnaires at the end of intervention, the $\mathrm{L} 1$ group confirmed a marked improvement in the subjective perception of the quality of life compared to the placebo group. Specifically, significantly higher scores were observed for various questions, such as "General health" in the L1 group (scoring from 40.02 to 47.5) compared to the placebo group (scoring from 37.86 to 37.97 ) (Fig. 3). In the same context, similar significant changes were observed for "Mental health" parameters, passing from 41.34 to 47.83 at

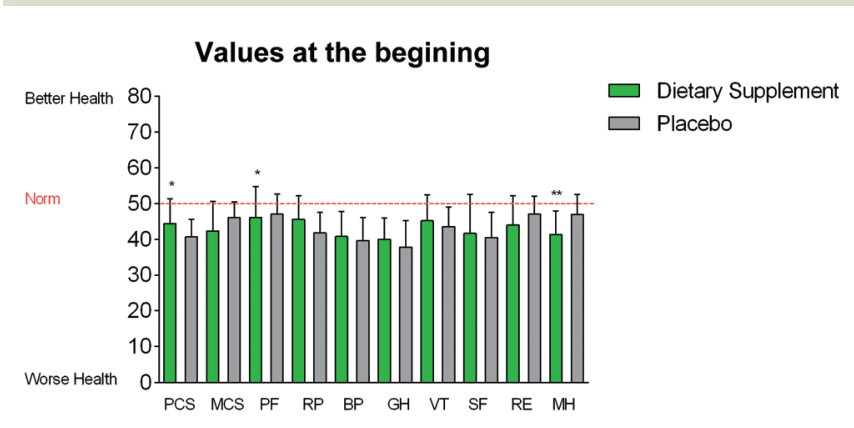

Values After Two Months

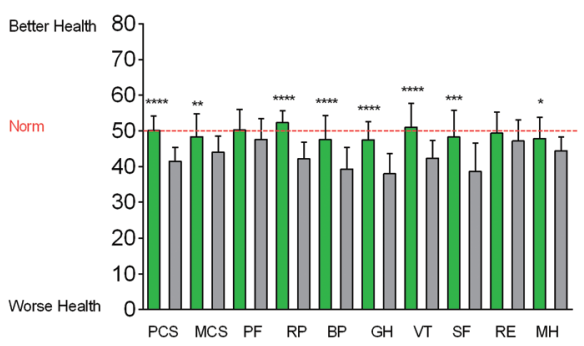

Fig. 3 Subjective scores for the SF-36 questionnaire in the dietary supplement group ( $L 1, n=25$ ) and placebo group (L2, $n=22)$, at the begining and two months of intervention. Data are represented using Health Outcomes Scoring Software and expressed as means \pm SD. Statistically significant differences were established between the two groups after one or two months after intra-group analysis as: ${ }^{*} p<0.05,{ }^{* *} p<0.01$, ${ }^{* * *} p<0.001,{ }^{* * * *} p<0.0001$. Physical Health Score (PCS), Mental Health score (MCS), Physical Functioning (PF), Role Physical (RP), Bodily Pain (BP), General Health (GH), Vitality (VT), Social Functioning (SF), Role Emotional (RE), Mental Health (MH). The higher scores of SF-36 correspond to the best state of health perception in each particular item. The 100 value corresponds to the higher score and the 50 value is the norm-based score (NBS). the end of intervention in the L1 group compared to the placebo group (scoring from 47.06 to 44.45). Finally, participants in the group consuming the polyphenolic supplement significantly improved in the psychological (ranging from 46.14 to 50.27 at the end of intervention) and pain parameters (scoring from 40.81 to 47.6 at the end of intervention) compared to the placebo group (Fig. 3).

\section{Determination of circulating appetite related peptides and hormones}

The data presented in Fig. 2 and 3 are subjective appreciations of satiety and health status. These positive sensations perceived by the subjects of the L1 group coincided with an improvement in anthropometric and vital parameters (Tables 1 and 2), although complementary analysis is required to confirm this hypothesis. To this end, the measurement of specific circulating peptides and obesity-related biomarkers can provide additional information related to the evolution of the pathology, suggesting at the same time putative mechanisms of action for the polyphenolic extracts assayed in this trial. In this vein, eight obesity-related markers were analyzed in plasma (Table 3). These peptides provide information regarding changes in adiposity, presence of insulin resistance and satiety.

It must be mentioned that certain appetite-related gut peptides (such as GLP-1, PYY and ghrelin) are secreted by the digestive track in the presence of nutrients. Since the plasma samples were obtained under fasting conditions, the levels determined for these particular gut hormones corresponded to basal values. Initially, baseline characteristics of the two groups were well matched and no significant differences were found (Table 3). Fibroblast growth factor 23 (FGF-23), a protein biomarker related to obesity and insulin resistance, showed a similar decrease in both groups, suggesting that the isocaloric diet was the most relevant factor in the reduction of this protein. Most of the other studied gut peptides and adipokines presented significant changes in the L1 group compared to the L2 group. The incretin GLP-1 increased significantly in the L1 group while it decreased in the L2 group. On the other hand, leptin significantly decreased in the L1 group at the end of intervention compared to the placebo group, where no significant changes were observed. In addition, resistin significantly decreased in the group consuming the dietary supplement, while no changes were observed in the L2 group. Finally, ghrelin presented a significant increase only in the placebo group, with no changes in the group that consumed the dietary supplement. In general, all the changes observed seem to give information that matches the positive evolution of the pathology in terms of weight reduction, blood pressure normalization, satiety control and optimal health status appreciation (Tables 1, 2 and Fig. 2, 3).

\section{Adverse events}

The consumption of the polyphenolic dietary supplement was well-tolerated. No adverse side effects were documented during the intervention period. 
Table 2 Differences with respect to baseline (beginning of the intervention) in anthropometric parameters and vital signs after one and two months expressed as means $\pm \mathrm{SE}$. Inter-group statistical analysis was performed at each period compared to the baseline. Significance was established at: ${ }^{*} p<0.05,{ }^{* *} p<0.01,{ }^{* * *} p<0.001,{ }^{* * *} p<0.0001$

\begin{tabular}{|c|c|c|c|c|}
\hline \multirow[b]{2}{*}{ Anthropometric parameters } & \multicolumn{2}{|c|}{ Differences after the first month } & \multicolumn{2}{|c|}{ Differences after two months } \\
\hline & Placebo (L2) & Supplement (L1) & Placebo (L2) & Supplement (L1) \\
\hline Body weight (kg) & $-1.44 \pm 0.27$ & $-2.46 \pm 0.28^{*}$ & $-2.08 \pm 0.30$ & $-3.48 \pm 0.40^{*}$ \\
\hline Body mass index $\left(\mathrm{kg} \mathrm{m}^{-2}\right)$ & $-0.63 \pm 0.14$ & $-1.00 \pm 0.15^{*}$ & $-0.83 \pm 0.12$ & $-1.37 \pm 0.16^{*}$ \\
\hline Arm circumference $(\mathrm{cm})$ & $0.03 \pm 0.03$ & $-0.11 \pm 0.06$ & $-0.22 \pm 0.23$ & $-0.32 \pm 0.08^{* *}$ \\
\hline $\mathrm{AC} 1(\mathrm{~cm})$ & $1.18 \pm 0.29$ & $-2.00 \pm 0.27^{*}$ & $-1.97 \pm 0.37$ & $-2.95 \pm 0.36$ \\
\hline $\mathrm{AC} 2(\mathrm{~cm})$ & $0.20 \pm 0.54$ & $-1.58 \pm 0.25^{* *}$ & $-0.80 \pm 0.55$ & $-2.57 \pm 0.34^{* *}$ \\
\hline Hip circumference $(\mathrm{cm})$ & $0.65 \pm 0.23$ & $-2.20 \pm 0.32^{* * *}$ & $-1.30 \pm 0.28$ & $-3.50 \pm 0.37^{* * * *}$ \\
\hline Triceps skinfold thickness (mm) & $-0.10 \pm 0.16$ & $-0.66 \pm 0.18^{*}$ & $-0.15 \pm 0.15$ & $-1.64 \pm 0.29 * * * *$ \\
\hline Biceps skinfold thickness (mm) & $-0.28 \pm 0.14$ & $-0.62 \pm 0.22$ & $-0.44 \pm 0.19$ & $-1.46 \pm 0.29^{* *}$ \\
\hline Abdominal skinfold thickness (mm) & $-0.33 \pm 0.36$ & $-1.73 \pm 0.37^{*}$ & $-1.33 \pm 0.29$ & $-2.84 \pm 0.53^{*}$ \\
\hline$\%$ Body fat & $0.29 \pm 0.06$ & $-0.56 \pm 0.06^{* *}$ & $-0.45 \pm 0.07$ & $-0.83 \pm 0.08^{* * *}$ \\
\hline \multicolumn{5}{|l|}{ Vital signs } \\
\hline Heart rate $(\mathrm{bpm})$ & $0.54 \pm 0.28$ & $-2.48 \pm 0.89^{* * * *}$ & $0.68 \pm 0.36$ & $-4.68 \pm 0.96^{* * * *}$ \\
\hline Systolic pressure (mm Hg) & $0.60 \pm 0.28$ & $-2.30 \pm 0.64^{* * * *}$ & $0.70 \pm 0.30$ & $-3.50 \pm 0.61 * * * *$ \\
\hline Diastolic pressure (mm Hg) & $0.32 \pm 0.43$ & $-2.12 \pm 0.39^{* * * *}$ & $0.00 \pm 0.50$ & $-3.92 \pm 0.63^{* * * *}$ \\
\hline
\end{tabular}

Abbreviations used: bpm, beats per minute.

Table 3 Levels of obesity and appetite-related gut peptides and hormones at the beginning (baseline) compared to the end (month 2) of intervention, expressed as means \pm SD. Significance was established at: ${ }^{*} p<0.05$. Statistical analysis was performed at two months compared to the baseline for each group

\begin{tabular}{|c|c|c|c|c|}
\hline & \multicolumn{2}{|l|}{ Placebo (L2) } & \multicolumn{2}{|c|}{ Supplement (L1) } \\
\hline FGF-23 $\left(\mathrm{pg} \mathrm{mL} \mathrm{mL}^{-1}\right)$ & $5.97 \pm 0.38$ & $5.67 \pm 0.09^{*}$ & $5.88 \pm 0,25$ & $5.72 \pm 0.18^{*}$ \\
\hline Ghrelin (pg mL $\left.\mathrm{mL}^{-1}\right)$ & $30.64 \pm 0.64$ & $33.74 \pm 3.48^{*}$ & $32.70 \pm 4.82$ & $32.89 \pm 3.23$ \\
\hline Insulin $\left(\mathrm{pg} \mathrm{mL}^{-1}\right)$ & $4.13 \pm 1.56$ & $3.674 \pm 2,03$ & $4.57 \pm 0.78$ & $4.74 \pm 0.55$ \\
\hline Leptin $\left(\mathrm{pg} \mathrm{mL}^{-1}\right)$ & $9669 \pm 6617$ & $5237 \pm 4886$ & $9772 \pm 4455$ & $4311 \pm 2304^{*}$ \\
\hline Resistin (pg mL $\left.{ }^{-1}\right)$ & $15593 \pm 6718$ & $10799 \pm 6624$ & $18101 \pm 6274$ & $12049 \pm 4022^{*}$ \\
\hline
\end{tabular}

\section{Discussion}

\section{Trial limitations}

The limitations that the study presents stem from the fasting conditions under which the plasma samples were obtained. The obtained values for gut hormones corresponded to basal levels. Postprandial complementary analysis needs to be performed in subsequent interventions for these particular hormones.

\section{External validity and applicability of the trial findings}

The female participants in this study were chosen from a Dietetics Shopping Centre in a southeastern Spanish city. Nevertheless, we can state that the results are applicable to participants in urban environments, taking into account the fact that lifestyles and eating habits are similar in most Spanish cities. On the other hand, other factors that can influence the study variables were not taken into account, as this study is centred exclusively on the consumption of particular polyphenol extracts and their effects on circulating satiety pep- tides as primary variables, and anthropometric and functional parameters used as secondary variables. Regarding the studied polyphenol extracts, the results would be valid under the aforementioned terms for the brand analysed.

\section{Discussion of results}

Despite the use of calorie restrictions through diet or increased caloric expenditure through exercise, many individuals still present enormous difficulties in losing weight and/or improving metabolic parameters. Considering the accumulated evidence on the effects of plant-derived polyphenols on obesity models, we proposed that some specifically designed nutraceuticals based on polyphenolic extracts may offer additional support for these particular patients in the management of obesity. ${ }^{17,20,23}$

Over the years, scientific evidence has shown that polyphenolic extracts from plant sources may be valuable tools to treat obesity. ${ }^{24}$ In this regard, our research at the pre-clinical and clinical level has shown the efficiency of the polyphenolic extracts from LC and HS. ${ }^{12-16}$ In the present intervention 
study and in the context of an isocaloric diet, we have observed that the group consuming the dietary supplement containing both polyphenolic extracts significantly reduced body weight, with a similar reduction in the body fat component compared with the placebo (Tables 1 and 2). This weight reduction was accompanied by the normalization of blood pressure and heart rate, which may be assigned to the reported capacity of the polyphenolic extracts from HS to reduce blood pressure and inflammatory plasma markers in humans. ${ }^{14,25}$ Because of the isocaloric diet, the placebo group also presented a similar tendency in the anthropometric parameters, but the differences at the end of intervention were statistically less prominent. Altogether, these data suggest that diet is an instrumental tool in weight reduction, but the dietary supplement seems to regulate other metabolic aspects further than those activated by calorie restriction and correct certain altered parameters.

The most intriguing effect observed by the participants who consumed the dietary supplement was a modulation of satiety. This parameter is instrumental in weight management, because it is the most effective parameter to achieve a consistent long-term weight loss and to stop body fat accumulation. Under normal circumstances, caloric restriction to reduce weight causes anxiety in the consumers, who often revert to compulsive intake, leading to weight regain. ${ }^{26}$ This situation decompensates diet balance and compromises weight reduction protocols. To this end, we have inquired trial participants about satiety using the validated VAS questionnaire. As a result, individuals consuming the dietary supplement reported significantly positive satiety scores compared to the placebo group (Fig. 2) and an improved perception of the quality of life through the SF-36 questionnaire (Fig. 3).

In short, energy balance is controlled by different components that are in charge of regulating appetite at the start and end of meals. These respond primarily to gastrointestinal signals that control satiety during meals, and to hormones secreted by the adipose tissue and hypothalamus that contribute to a more balanced feeding behavior in the long term. ${ }^{2}$ Consequently, a selection of peptides and hormones that regulate hunger and satiety and adipokines was analyzed, which are generally altered in overweight individuals. These include: FGF-23, GLP-1, ghrelin, insulin, leptin, C-peptide, PYY and resistin. It is important to note that satiety is a complex mechanism, mediated by a variety of orexigenic and anorexigenic signals that are integrated in the hypothalamus. ${ }^{27}$ Therefore, the analysis of only one or a few peptides may not be sufficient to understand satiety regulation. Furthermore, for a diet-induced weight loss, satiety is not the only factor involved, as fat reduction and metabolic normalization also play a role in regulating these peptides due to the variation of adiposity. Therefore, in this particular study, the changes observed in the analyzed gut hormones and obesity-related biomarkers must be taken in a more generalized, simplified context where satiety could be a supporting component for weight reduction, but where other parameters may also be involved.
In our study, blood samples were obtained under fasting conditions. Therefore, the biomarkers were analyzed in a "hunger" state, and the results must be interpreted accordingly (Table 3). For example, GLP-1 is an anorexigenic incretin produced by the intestinal L-cells that stimulates insulin secretion while also inducing satiety. ${ }^{28}$ The results shown in Table 3 indicated that the group taking the polyphenolic dietary supplement presented higher levels of this cytokine after two months compared to the placebo group, most likely contributing to the satiety effect perceived by the consumers (Fig. 2). The other gastrointestinal peptide (PYY) did not show significant differences in any of the groups by comparing the beginning and the end of the intervention. On the other hand, the orexigenic hormone ghrelin is secreted by the stomach and induces appetite. ${ }^{24}$ This cytokine was significantly increased only in the placebo group, which could contribute to increased food intake and stimulate appetite after calorie restriction. In normal weight individuals, plasma ghrelin concentrations rise during fasting and drop with meal ingestion proportional to the calorie content. However overweight individuals do not display the same suppression of ghrelin in response to calorie ingestion and weight loss led to increased plasma level in overweight individuals as a compensatory response. ${ }^{29-31}$ Other studies have corroborated that the intravenous administration of ghrelin increases both appetite and food intake. ${ }^{32}$ Our results of higher ghrelin levels at the end of the study for the placebo group clearly indicate a higher hunger sensation for this group compared to the group having the supplement, which could explain, at least in part, the higher satiety results observed in the hunger-related sensation analysis for the intervention group compared to the placebo group (Fig. 2).

Leptin can be considered as a lipostatic hormone mainly secreted by adipose tissue. Leptin exerts a variety of effects on target tissues, adapting specific functions to the amount of fat present in the organism. ${ }^{33}$ In addition, leptin decreases appetite by stimulating the secretion of anorexigenic peptides by the hypothalamus, increasing basal metabolism, reducing lipogenesis and augmenting lipolysis to produce energy. ${ }^{2}$ In the present study, the placebo group did not show significant changes in leptin values, while the group consuming the dietary supplement displayed a significant reduction in plasmatic leptin levels. Although high leptin levels induce satiety, the loss of body weight (mainly adipose tissue) observed by the participants may be accompanied by a decrease in leptin secretion by adipocytes. These results about leptin may be difficult to reconcile on the basis of satiety control only and should be interpreted in relation to the complex variety of functions exerted by this peptide. ${ }^{33}$ In fact, it is well established that many overweight individuals show leptin resistance i.e., higher levels of leptin in blood are necessary to exert its effect on target tissues. In addition, we have also observed a decrease of leptin by plant-derived polyphenols in correlation to decreased triglyceride content in a hypertrophic adipocyte model. ${ }^{12}$ We postulate that the normalization of leptin levels achieved by the weight reduction strategy could contribute to modulate the satiety sensation and the function of other leptin 
targets more efficiently. However, as previously mentioned, a post-prandial analysis would have provided more accurate results of this adipokine. In this context, resistin is generally increased in overweight individuals, giving rise to insulin resistance. ${ }^{34}$ The expression level of this adipokine was significantly lowered in the group consuming the polyphenolic supplement, but not in the placebo group. This could favor leptin's effect by controlling satiety as well as other functions. Moreover, FGF-23 was originally considered to be implicated in bone metabolism, but recently it has been shown to also play a role in obesity and cardiovascular diseases. ${ }^{35}$ In the present study, FGF-23 was significantly decreased in both groups, suggesting that the balanced diet could play a more prominent role than the polyphenolic extract in the expression of this growth factor. Finally, insulin and C-peptide did not show significant changes in both groups.

In our intervention study, overweight participants from both groups followed an isocaloric diet for two months; so, in response to a first period of weight loss, the calorie restriction should have led to a compensatory adaptation phenomenon governed by the hypothalamus, i.e. "energy gap", by increasing hunger and reducing energy expenditure, as reported (Fig. 4). ${ }^{7-9}$ According to this concept, upon dietary restriction, individuals losing weight increase orexigenic hormones and peptides (ghrelin) $^{36,37}$ and decrease anorexigenic hormones (GLP-1) ${ }^{38}$ in order to increase hunger and to reduce energy expenditure which triggers weight regain. In response to diet, leptin and resistin also decrease, due presumably to reduced adiposity, leading to increased hunger and subsequently to weight regain.

Interestingly, the results of our trial can be explained on the basis of counteracting the "energy gap" phenomenon. ${ }^{7}$ In our trial these two hormones, leptin and resistin, decreased in both groups, most probably in correlation to decreased adiposity. Contrary to the expected behavior upon the calorie restriction situation, in the group having the dietary supplement for two months, we could observe an increase in anorexigenic hormones (GLP-1) and a normalization of orexigenic hormones (ghrelin). Therefore, we propose that the consumption of the dietary supplement counteracted the effects associated with dietary restriction, promoting anorexigenic hormones and decreasing orexigenic hormones and restoring the balance between hunger and energy (Fig. 4). In a similar way, appropriate dietary interventions that reduce hunger (increasing dietary protein and fiber) and increase energy expenditure (exercise) are proposed for a long-term weight loss maintenance, narrowing the energy gap. ${ }^{7}$

Evidence derived from our research and those of others have proposed the activation of the energy sensor AMPK by polyphenols as a therapeutic target for obesity, ${ }^{18,19,39}$ through the modulation of lipid accumulation, ${ }^{17-19}$ the inhibition of oxidative stress and the blockage of inflammatory adipokine secretion. ${ }^{12,25,40}$ According to these pieces of evidence, we hypothesize that the polyphenolic extracts in the combination could help to reduce adipose fat mass, by modulating AMPK activity in these tissues, with the concomitant normalization of the secreted levels of leptin and resistin. However, further research may be required to confirm the role of the combination of polyphenolic extracts in gastrointestinal peptide secretion and to prove the AMPK-activating properties in vivo.

We also postulate that the dietary supplement, besides regulating satiety, might also modulate some components of the TDEE, probably through its capability to modulate AMPK in

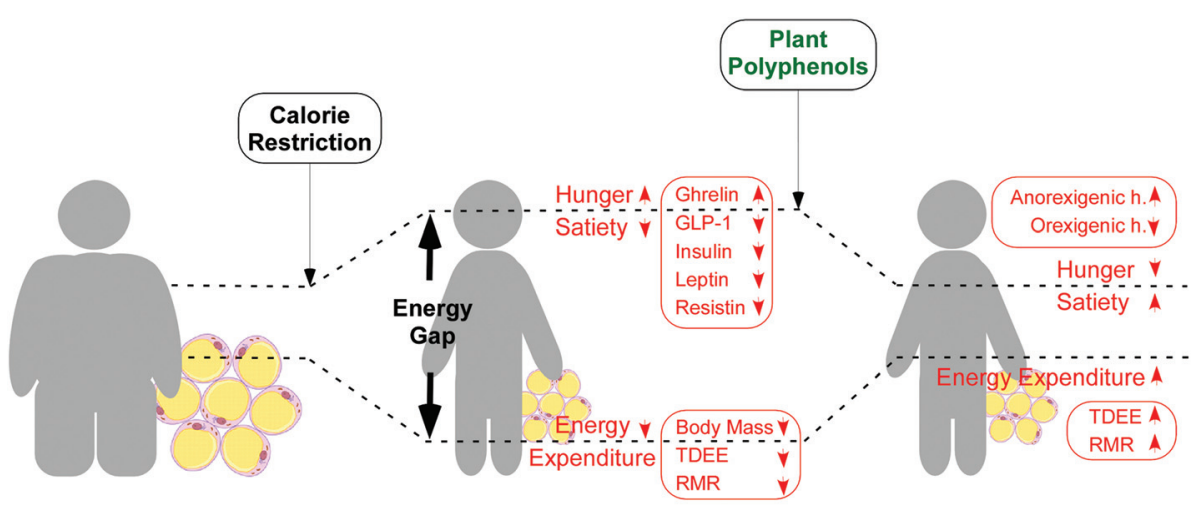

Before Weight Loss

After Weight Loss

Long-Term Weight Loss

Fig. 4 Modulation of the energy gap by diet restriction and polyphenol supplementation. In response to calorie restriction and the subsequent weight loss, discordance between nutrient ingestion and energy expenditure appears, i.e. the "energy gap". This metabolic situation leads to the brain triggering a compensatory hormonal response that reflects both appetite-related hormones and peptide changes and energy expenditure adaptation. On one side, orexigenic hormones are enhanced resulting in increased hunger. On the expenditure side, weight loss and reduced adiposity cause a metabolic adaptation consisting of the reduction of total daily energy expenditure (TDEE), most probably due to a reduced resting metabolic rate (RMR) and decreased thermic effect of food. The increased hunger and reduced energy expenditure give rise to prompt weight regain. We propose that the consumption of some plant polyphenols counteracts these effects by promoting anorexigenic hormones and decreasing orexigenic hormones and restores the balance between hunger and energy. This may enable long-term weight loss maintenance by narrowing the energy gap upon calorie restriction (adapted from Melby, Paris, Foright, \& Peth, 2017). 
different tissues. Indeed, it has also been shown that the activation of AMPK has pleiotropic effects in multiple tissues. ${ }^{41,42}$ AMPK activation in most tissues (liver, adipose tissue and muscle) promotes increased fatty acid oxidation, glucose uptake and glycolysis as well as the inhibition of fatty acid, cholesterol and glycogen synthesis. Paradoxically, the activation of hypothalamic AMPK led to increased food intake. ${ }^{41,43}$ Whether metabolites of polyphenols derived from the dietary supplement can pass through the haematoencephalic barrier and reach AMPK directly at the hypothalamus still needs to be verified.

With regard to the putative metabolites capable of undertaking these effects, previous evidence lead us to propose some phenylpropanoids from $L$. citriodora and flavonol derivatives from $H$. sabdariffa. ${ }^{44-47}$ In any case, these hypotheses need to be verified in further animal trials specifically designed for such measurements.

In conclusion, the consumption of $500 \mathrm{mg}^{-1 a y}{ }^{-1}$ of the combination of Hibiscus and Lippia polyphenolic extracts in the context of an isocaloric diet for two months in overweight subjects confirmed significant reductions of body fat, blood pressure and heart rate and a more positive perception in their overall health status compared to the placebo group. In addition, trial participants exhibited a decrease in hunger and appetite and a lower attraction for fatty, sweet and salty food compared to the placebo group, concomitantly with a reduction in circulating resistin and normalization of leptin expression, while regulating ghrelin and GLP-1 levels (which were higher and lower in the placebo group, respectively). Altogether, the results of the present study demonstrate that polyphenols are capable of modulating adipohormones and gut peptide expression and control satiety and hunger states, with AMPK modulation in different tissues as a putative target, although the underlying mechanism is still unknown.

\section{Conflicts of interest}

This study was partially funded by Monteloeder S. L. Monteloeder was involved in the design of the study protocol and provided the test product samples. Employees of the sponsor were not involved in data analysis. The manuscript was prepared by Maria Herranz, Enrique Roche and Vicente Micol. Monteloeder was permitted to review the manuscript and suggest changes, but the final decision on content was exclusively retained by the corresponding author. Dr Vicente Micol is the guarantor for this article and takes responsibility for the integrity of the work as a whole. NC works for Monteloeder S. L.

\section{Abbreviations}

\section{LC Lippia citriodora \\ HS Hibiscus sabdariffa}

AMPK AMP-activated protein kinase
TDEE Total daily energy expenditure

RMR Resting metabolic rate

AC Abdominal circumferences

BMI Body mass index

VAS Validated visual analogue scale

PYY Gastrointestinal peptide

FGF-23 Fibroblast growth factor 23

GLP-1 Glucagon-like peptide-1

\section{Acknowledgements}

This work has been funded by the following grants: AGL201129857-C03-03 from the Spanish Ministry of Science and Innovation; Grant AGL2015-67995-C3-1-R and PTQ-14-07243 from the Spanish Ministry of Economy and Competitiveness (MINECO); PROMETEO/2012/007, PROMETEO/2016/006, ACIF/ 2013/064 and ACIF/2016/230 from Generalitat Valenciana; and CB12/03/30038 grant from CIBER (Fisiopatologia de la Obesidad y la Nutricion, CIBERobn, Instituto de Salud Carlos III, Spain). We also thank the IDI-20150608 AVANZAS project from the Spanish Centre for the Development of Industrial Technology (CDTI, MINECO). This article has been partially funded by a H2020 SME Phase II project, grant number 783838 .

\section{References}

1 M. Luna-Luna, A. Medina-Urrutia, G. Vargas-Alarcon, F. Coss-Rovirosa, J. Vargas-Barron and O. Perez-Mendez, Adipose Tissue in Metabolic Syndrome: Onset and Progression of Atherosclerosis, Arch. Med. Res., 2015, 46, 392-407.

2 K. Timper and J. C. Bruning, Hypothalamic circuits regulating appetite and energy homeostasis: pathways to obesity, Dis. Models \& Mech., 2017, 10, 679-689.

3 C. Drenowatz, Reciprocal Compensation to Changes in Dietary Intake and Energy Expenditure within the Concept of Energy Balance, Adv. Nutr., 2015, 6, 592-599.

4 A. Vegiopoulos, M. Rohm and S. Herzig, Adipose tissue: between the extremes, EMBO J., 2017, 36, 1999-2017.

5 G. Asghari, P. Mirmiran, E. Yuzbashian and F. Azizi, A systematic review of diet quality indices in relation to obesity, Br. J. Nutr., 2017, 117, 1055-1065.

6 J. Vairavamurthy, L. J. Cheskin, D. L. Kraitchman, A. Arepally and C. R. Weiss, Current and cutting-edge interventions for the treatment of obese patients, Eur. J. Radiol., 2017, 93, 134-142.

7 C. L. Melby, H. L. Paris, R. M. Foright and J. Peth, Attenuating the Biologic Drive for Weight Regain Following Weight Loss: Must What Goes Down Always Go Back Up?, Nutrients, 2017, 9, DOI: 10.3390/nu9050468.

8 Z. J. Zhao, Y. A. Liu, J. Y. Xing, M. L. Zhang, X. Y. Ni and $\mathrm{J}$. Cao, The role of leptin in striped hamsters subjected to food restriction and refeeding, Dongwuxue Yanjiu, 2014, 35, 262-271. 
9 P. Sumithran and J. Proietto, The defence of body weight: a physiological basis for weight regain after weight loss, Clin. Sci., 2013, 124, 231-241.

10 M. Rosenbaum, J. Hirsch, D. A. Gallagher and R. L. Leibel, Long-term persistence of adaptive thermogenesis in subjects who have maintained a reduced body weight, Am. J. Clin. Nutr., 2008, 88, 906-912.

$11 \mathrm{~J}$. J. Reho and K. Rahmouni, Oxidative and inflammatory signals in obesity-associated vascular abnormalities, Clin. Sci., 2017, 131, 1689-1700.

12 M. Herranz-Lopez, S. Fernandez-Arroyo, A. Perez-Sanchez, E. Barrajon-Catalan, R. Beltran-Debon, J. A. Menendez, C. Alonso-Villaverde, A. Segura-Carretero, J. Joven and V. Micol, Synergism of plant-derived polyphenols in adipogenesis: perspectives and implications, Phytomedicine, 2012, 19, 253-261.

13 J. Joven, E. Espinel, A. Rull, G. Aragones, E. RodriguezGallego, J. Camps, V. Micol, M. Herranz-Lopez, J. A. Menendez, I. Borras, A. Segura-Carretero, C. AlonsoVillaverde and R. Beltran-Debon, Plant-derived polyphenols regulate expression of miRNA paralogs miR-103/107 and miR-122 and prevent diet-induced fatty liver disease in hyperlipidemic mice, Biochim. Biophys. Acta, 2012, 1820, 894-899.

14 J. Joven, I. March, E. Espinel, S. Fernandez-Arroyo, E. Rodriguez-Gallego, G. Aragones, R. Beltran-Debon, C. Alonso-Villaverde, L. Rios, V. Martin-Paredero, J. A. Menendez, V. Micol, A. Segura-Carretero and J. Camps, Hibiscus sabdariffa extract lowers blood pressure and improves endothelial function, Mol. Nutr. Food Res., 2014, 58, 1374-1378.

15 S. Fernández-Arroyo, I. C. Rodríguez-Medina, R. BeltránDebón, F. Pasini, J. Joven, V. Micol, A. Segura-Carretero and A. Fernández-Gutiérrez, Quantification of the polyphenolic fraction and in vitro antioxidant and in vivo anti-hyperlipemic activities of Hibiscus sabdariffa aqueous extract, Food Res. Int., 2011, 44, 1490-1495.

16 M. Herranz-López, E. Barrajón-Catalán, A. SeguraCarretero, J. A. Menéndez, J. Joven and V. Micol, Lemon verbena (Lippia citriodora), polyphenols alleviate obesityrelated disturbances in hypertrophic adipocytes through AMPK-dependent mechanisms, Phytomedicine, 2015, 22, 605-614.

17 M. Herranz-Lopez, M. Olivares-Vicente, J. A. Encinar, E. Barrajon-Catalan, A. Segura-Carretero, J. Joven and V. Micol, Multi-Targeted Molecular Effects of Hibiscus sabdariffa Polyphenols: An Opportunity for a Global Approach to Obesity, Nutrients, 2017, 9, DOI: 10.3390/nu9080907.

18 E. Barrajon-Catalan, M. Herranz-Lopez, J. Joven, A. SeguraCarretero, C. Alonso-Villaverde, J. A. Menendez and V. Micol, Molecular promiscuity of plant polyphenols in the management of age-related diseases: far beyond their antioxidant properties, Adv. Exp. Med. Biol., 2014, 824, 141159.

19 J. Joven, A. Rull, E. Rodriguez-Gallego, J. Camps, M. RieraBorrull, A. Hernandez-Aguilera, V. Martin-Paredero,
A. Segura-Carretero, V. Micol, C. Alonso-Villaverde and J. A. Menendez, Multifunctional targets of dietary polyphenols in disease: a case for the chemokine network and energy metabolism, Food Chem. Toxicol., 2013, 51, 267-279.

20 J. Joven, V. Micol, A. Segura-Carretero, C. Alonso-Villaverde, J. A. Menéndez, G. Aragonès, E. Barrajón-Catalán, R. Beltrán-Debón, J. Camps, S. Cufí, S. Fernández-Arroyo, A. Fernández-Gutiérrez, E. Guillén, M. Herranz-López, I. Iswaldi, J. Lozano-Sánchez, B. Martin-Castillo, C. Oliveras-Ferraros, A. Pérez-Sánchez, E. RodríguezGallego, A. Rull, D. Saura and A. Vázquez-Martín, Polyphenols and the Modulation of Gene Expression Pathways: Can We Eat Our Way Out of the Danger of Chronic Disease?, Crit. Rev. Food Sci. Nutr., 2014, 54, 9851001.

21 A. Weltman, S. Levine, R. L. Seip and Z. V. Tran, Accurate assessment of body composition in obese females, Am. J. Clin. Nutr., 1988, 48, 1179-1183.

22 A. Boyum, Separation of White Blood Cells, Nature, 1964, 204, 793-794.

23 S. Camacho, S. Michlig, C. de Senarclens-Bezencon, J. Meylan, J. Meystre, M. Pezzoli, H. Markram and J. le Coutre, Anti-obesity and anti-hyperglycemic effects of cinnamaldehyde via altered ghrelin secretion and functional impact on food intake and gastric emptying, Sci. Rep., 2015, 5, 7919.

24 S. Wang, N. Moustaid-Moussa, L. Chen, H. Mo, A. Shastri, R. Su, P. Bapat, I. Kwun and C. L. Shen, Novel insights of dietary polyphenols and obesity, J. Nutr. Biochem., 2014, 25, 1-18.

25 R. Beltran-Debon, C. Alonso-Villaverde, G. Aragones, I. Rodriguez-Medina, A. Rull, V. Micol, A. Segura-Carretero, A. Fernandez-Gutierrez, J. Camps and J. Joven, The aqueous extract of Hibiscus sabdariffa calices modulates the production of monocyte chemoattractant protein-1 in humans, Phytomedicine, 2010, 17, 186-191.

26 C. Ural, H. Belli, M. Akbudak, A. Solmaz, Z. D. Bektas and F. Celebi, Relation of binge eating disorder with impulsiveness in obese individuals, World J. Psychiatr., 2017, 7, 114120.

27 T. Amin and J. G. Mercer, Hunger and Satiety Mechanisms and Their Potential Exploitation in the Regulation of Food Intake, Curr. Obes. Rep., 2016, 5, 106-112.

28 T. A. Lutz, Gut hormones such as amylin and GLP-1 in the control of eating and energy expenditure, Int. J. Obes. Suppl., 2016, 6, S15-S21.

29 C. W. le Roux, M. Patterson, R. P. Vincent, C. Hunt, M. A. Ghatei and S. R. Bloom, Postprandial plasma ghrelin is suppressed proportional to meal calorie content in normal-weight but not obese subjects, J. Clin. Endocrinol. Metab., 2005, 90, 1068-1071.

30 P. J. English, M. A. Ghatei, I. A. Malik, S. R. Bloom and J. P. Wilding, Food fails to suppress ghrelin levels in obese humans, J. Clin. Endocrinol. Metab., 2002, 87, 2984.

31 D. E. Cummings, J. Q. Purnell, R. S. Frayo, K. Schmidova, B. E. Wisse and D. S. Weigle, A preprandial rise in plasma 
ghrelin levels suggests a role in meal initiation in humans, Diabetes, 2001, 50, 1714-1719.

32 N. M. Neary, C. J. Small, A. M. Wren, J. L. Lee, M. R. Druce, C. Palmieri, G. S. Frost, M. A. Ghatei, R. C. Coombes and S. R. Bloom, Ghrelin increases energy intake in cancer patients with impaired appetite: acute, randomized, placebo-controlled trial, J. Clin. Endocrinol. Metab., 2004, 89, 2832-2836.

33 J. S. Flier and E. Maratos-Flier, Leptin's Physiologic Role: Does the Emperor of Energy Balance Have No Clothes?, Cell Metab., 2017, 26, 24-26.

34 X. Huang and Z. Yang, Resistin's, obesity and insulin resistance: the continuing disconnect between rodents and humans, J. Endocrinol. Invest., 2016, 39, 607-615.

35 X. Hu, X. Ma, Y. Luo, Y. Xu, Q. Xiong, X. Pan, Y. Xiao, Y. Bao and W. Jia, Associations of serum fibroblast growth factor 23 levels with obesity and visceral fat accumulation, Clin. Nutr., 2018, 37, 223-228.

36 A. B. Crujeiras, E. Goyenechea, I. Abete, M. Lage, M. C. Carreira, J. A. Martinez and F. F. Casanueva, Weight regain after a diet-induced loss is predicted by higher baseline leptin and lower ghrelin plasma levels, J. Clin. Endocrinol. Metab., 2010, 95, 5037-5044.

37 D. E. Cummings, D. S. Weigle, R. S. Frayo, P. A. Breen, M. K. Ma, E. P. Dellinger and J. Q. Purnell, Plasma ghrelin levels after diet-induced weight loss or gastric bypass surgery, N. Engl. J. Med., 2002, 346, 1623-1630.

38 P. Sumithran, L. A. Prendergast, E. Delbridge, K. Purcell, A. Shulkes, A. Kriketos and J. Proietto, Long-term persistence of hormonal adaptations to weight loss, N. Engl. J. Med., 2011, 365, 1597-1604.

39 D. Carling, AMPK signalling in health and disease, Curr. Opin. Cell Biol., 2017, 45, 31-37.

40 S. Furukawa, T. Fujita, M. Shimabukuro, M. Iwaki, Y. Yamada, Y. Nakajima, O. Nakayama, M. Makishima,
M. Matsuda and I. Shimomura, Increased oxidative stress in obesity and its impact on metabolic syndrome, J. Clin. Invest., 2004, 114, 1752-1761.

41 B. Kola, Role of AMP-activated protein kinase in the control of appetite, J. Neuroendocrinol., 2008, 20, 942-951.

42 B. Kola, M. Boscaro, G. A. Rutter, A. B. Grossman and M. Korbonits, Expanding role of AMPK in endocrinology, Trends Endocrinol. Metab., 2006, 17, 205-215.

43 M. Lopez, R. Nogueiras, M. Tena-Sempere and C. Dieguez, Hypothalamic AMPK: a canonical regulator of wholebody energy balance, Nat. Rev. Endocrinol., 2016, 12, 421432.

44 R. Quirantes-Pine, M. Herranz-Lopez, L. Funes, I. BorrasLinares, V. Micol, A. Segura-Carretero and A. FernandezGutierrez, Phenylpropanoids and their metabolites are the major compounds responsible for blood-cell protection against oxidative stress after administration of Lippia citriodora in rats, Phytomedicine, 2013, 20, 1112-1118.

45 M. Herranz-Lopez, I. Borras-Linares, M. Olivares-Vicente, J. Galvez, A. Segura-Carretero and V. Micol, Correlation between the cellular metabolism of quercetin and its glucuronide metabolite and oxidative stress in hypertrophied 3 T3-L1 adipocytes, Phytomedicine, 2017, 25, 25-28.

46 S. Fernandez-Arroyo, M. Herranz-Lopez, R. Beltran-Debon, I. Borras-Linares, E. Barrajon-Catalan, J. Joven, A. Fernandez-Gutierrez, A. Segura-Carretero and V. Micol, Bioavailability study of a polyphenol-enriched extract from Hibiscus sabdariffa in rats and associated antioxidant status, Mol. Nutr. Food Res., 2012, 56, 1590-1595.

47 M. Olivares-Vicente, E. Barrajon-Catalan, M. HerranzLopez, A. Segura-Carretero, J. Joven, J. A. Encinar and V. Micol, Plant-derived polyphenols in human health: biological activity, metabolites and putative molecular targets, Curr. Drug. Metab., 2018, 19, DOI: 10.2174/ 1389200219666180220095236. 